\title{
A dynamic modification to sneutrino chaotic inflation
}

\section{Abhijit Kumar Saha and Arunansu Sil}

Department of Physics, Indian Institute of Technology,

Guwahati, Assam 781039, India

E-mail: abhijit.saha@iitg.ernet.in, asil@iitg.ernet.in

ABSTRACT: We consider a right-handed scalar neutrino as the inflaton which carries a gravitational coupling with a supersymmetric QCD sector responsible for breaking supersymmetry dynamically. The framework suggests an inflaton potential which is a deformed version of the quadratic chaotic inflation leading to a flatter potential. We find that this deformation results a sizable tensor to scalar ratio which falls within the allowed region by PLANCK 2015. At the same time supersymmetry breaking at the end of inflation can naturally be induced in this set-up. The symmetries required to construct the framework allows the neutrino masses and mixing to be of right order.

Keywords: Supersymmetry Breaking, Beyond Standard Model, Cosmology of Theories beyond the SM

ArXiv ePrint: 1509.00218 


\section{Contents}

1 Introduction 1

2 Standard sneutrino chaotic inflation in supergravity 3

3 SQCD sector and supersymmetry breaking in a metastable vacuum 5

4 Interaction between neutrino and SQCD sectors $\quad 7$

5 Modified chaotic potential and its implications to inflationary dynamics 8

6 Results 10

7 Dynamics after inflation 12

8 Dynamical breaking of $\mathrm{U}(1)_{R} \quad 13$

9 Neutrino masses and mixing $\quad 14$

10 Reheating 16

$\begin{array}{ll}11 \text { Conclusion } & 17\end{array}$

$\begin{array}{ll}\text { A Finding the root of } \sigma & 18\end{array}$

B $R$ charges of various fields $\quad 19$

\section{Introduction}

The inflationary paradigm is well accepted as a successful theory of the early universe with its interpretation of several shortcomings of the Bigbang cosmology in an economic way. This hypothesis is further strengthened by its prediction on the primordial perturbations that leads to the striking agreement with the observation of the cosmic microwave background (CMB) spectrum. Among the various models of inflation, large field inflation models receive a lot of attention these days, particularly after the claim of BICEP2 [1], due to their ability to produce a large tensor to scalar ratio $(r)$. Although this particular claim is shadowed by the recent release of PLANCK 2015 [2,3] data which provides an upper bound on $r$ as $r \simeq 0.11$, large field inflation remains as an interesting possibility to explore in view of future search for observing tensor perturbations in CMB by PLANCK 2015 and other experiments with a greater accuracy.

The chaotic inflation in supergravity (SUGRA) as proposed in $[4,5]$ having a scalar potential of the form $V=\frac{1}{2} m^{2} \chi^{2}$ is possibly the simplest scenario of this sort of inflation 
model [6] with its prediction of $r$ as 0.13 . The mass scale $m$ turns out to be of order $10^{13} \mathrm{GeV}$. Within this large-field inflationary scenario, the inclusion of supergravity induces a particular problem (known as the $\eta$ problem). This is caused by the field value of the inflaton $(\chi)$ during inflation, which exceeds the reduced Planck scale $M_{P} \simeq 2.4 \times 10^{18} \mathrm{GeV}$, and thereby spoiling the required degree of flatness of the inflationary potential through the Planck-suppressed operators. In [4], a shift symmetric Kähler potential associated with the inflaton field was considered to cure this problem. However the PLANCK 2015 [2, 3] suggests a modification of the standard chaotic inflation as it barely enters into the $2 \sigma$ range of $n_{s}-r$ (spectral index vs. tensor to scalar ratio) plot. Analyses [7, 8] with PLANCK 2013 [9] data followed by the BICEP2 [1] suggest modification of the Kähler potential by introducing a shift-symmetry breaking term. A general deformation of the chaotic superpotential by including higher order terms with very small coefficients has been exercised in $[10,11]$. All these analyses would be further restricted by the recent release of PLANCK 2015 [2, 3] data.

It has long been exercised how an inflationary scenario can be linked with the particle physics framework. In this regard, neutrino physics can provide an interesting possibility. It is well known that the smallness of the light neutrino mass $\left(m_{\nu}\right)$ can be explained by type-I seesaw mechanism which enforces the inclusion of heavy right handed $(\mathrm{RH})$ neutrinos $(N)$. In a supersymmetric theory, the close proximity of the mass scale of these heavy RH fields and their superpartners (sneutrinos) with the mass parameter $m$ involved in the chaotic inflationary potential as mentioned before, suggests that the sneutrino can actually play the role for the inflaton. Indeed, it was shown [12-16] that the standard chaotic inflation can actually be realized including them. Another interesting aspect of a supersymmetric model of inflation is its relation with supersymmetry breaking. From the completeness point of view, a supersymmetric structure of an inflationary scenario demands a realization of supersymmetry breaking at the end of inflation. Though during inflation, the vacuum energy responsible for inflation breaks supersymmetry (at a large scale of order of energy scale of inflation), as the inflaton field finally rolls down to a global supersymmetric minimum, it reduces to zero vacuum energy, and thereby no residual supersymmetry breaking remains.

In this work, our purpose is two fold; one is to modify the standard sneutrino chaotic inflation so as to satisfy the PLANCK $2015[2,3]$ results and other is to accommodate supersymmetry breaking at the end of inflation. We consider two sectors namely (i) the inflation sector and (ii) the supersymmetry breaking sector. The inflation sector is part of the neutrino sector consisting of three RH neutrino superfields. There will be a role for another sneutrino during inflation, which will be unfolded as we proceed. We identify the scalar field responsible for inflation to be associated with one of these three fields. The scalar potential resembles the standard chaotic inflation in the supergravity framework assisted with the shift symmetric Kähler potential. The superpotential involving the RH neutrino responsible for inflation breaks this shift symmetry softly. We have argued the smallness associated with this shift symmetry breaking parameter by introducing a spurion field. In this excercise, we also consider discrete symmetries to forbid unwanted terms. For the supersymmetry breaking sector, we consider the Intriligator-Seiberg-Shih (ISS) model [17] 
of breaking supersymmetry dynamically in a metastable vacuum. This sector is described by a supersymmetric gauge theory and henceforth called the SQCD sector. These two sectors can have a gravitational coupling which in turn provides a dynamical deformation of the standard chaotic inflation. Again the coupling strength between these two sectors can be naturally obtained through another spurion. As the inflaton field approaches its minimum once the inflation is over, this interaction term becomes insignificant and finally the two sectors are effectively decoupled. However the hidden SQCD sector fields stabilize in metastable vacuum, hence supersymmetry breaking is achieved as a remnant of inflation. Earlier attempts in connecting the inflation and ISS type supersymmetry breaking can be found in [18-21]. A global $\mathrm{U}(1)_{R}$ symmetry plays a pivotal role in shaping the ISS model of dynamic supersymmetry breaking. Once the supersymmetry is broken in the hidden SQCD sector, the effective supersymmetry breaking scale in the Standard Model sector is assumed here to be developed by the gauge mediation mechanism. To materialize this, the ISS model requires a modification for breaking $\mathrm{U}(1)_{R}$. In this context we follow the proposal in [22] and show that this can easily be adopted in our set-up. Furthermore, as the RH neutrino superfields are part of the inflation sector, which obeys the same $\mathrm{U}(1)_{R}$ symmetry, their $\mathrm{U}(1)_{R}$ charges are already fixed. The same $\mathrm{RH}$ neutrinos also contribute to the light neutrino mass matrix through type-I seesaw mechanism. We find that the $\mathrm{U}(1)_{R}$ charges of various fields involved along with their charges under the discrete symmetries imposed can actually predict an inverted hierarchy of neutrino masses. We provide an estimate of reheating temperature in this context and also comment on leptogenesis.

Below in section 2, we briefly discuss the standard chaotic inflation in the supergravity framework. Then we will discuss about the ISS model of dynamic supersymmetry breaking in section 3 followed by the role of interaction term between the two sectors in section 4 . The dynamics of the fields during and after inflation are discussed in section 5 and 7 respectively. The prediction for this modified chaotic inflation are presented in section 6 . In section 8, we have shown that a deformation to the SQCD sector can be achieved which is related to the $\mathrm{U}(1)_{R}$ symmetry breaking. In section 9 , we discuss the implication of neutrino masses and mixing that comes out of the present set-up. We comment on the reheating temperature and leptogenesis in section 10 . Finally we conclude in section 11.

\section{Standard sneutrino chaotic inflation in supergravity}

We start this section by reviewing some of the features of the standard chaotic inflation in supergravity where the scalar partner of a $\mathrm{RH}$ neutrino (say $N_{1}$ among the three $\mathrm{RH}$ superfields $N_{i=1,2,3}$ involved in type-I seesaw for generating light neutrino mass) serves the role of inflaton. Sneutrino chaotic inflation $[12,13,15,16]$ gains much attention from the perspective of particle physics involvement. Mass of the inflaton and in turn mass of that particular RH neutrino (in the supersymmetric limit) can be fixed by the magnitude of curvature perturbation spectrum in this theory. In $\mathcal{N}=1$ SUGRA, the superpotential is considered to be

$$
W_{N}=m N_{1} N_{2}
$$


along with the Kähler Potential ${ }^{1}$

$$
K_{N}=\left|N_{2}\right|^{2}-\frac{\left(N_{1}-N_{1}^{\dagger}\right)^{2}}{2}
$$

Note that a shift symmetry, $N_{1} \rightarrow N_{1}+\mathrm{C}$, where $\mathrm{C}$ is real having mass dimension unity, is imposed on the Kähler potential, whereas the superpotential breaks it. Thus the parameter $m$ can be regarded as a shift-symmetry breaking parameter.

The parameter $m$ being much smaller than $M_{P}$, the term in the superpotential $W_{N}$ would be natural in 't Hooft's sense [23] of increased symmetry in the limit $m \rightarrow 0$. The smallness associated with $m$ can be explained with the introduction of a spurion field $z_{1}$ as shown in [5]. Also the higher order shift symmetry breaking terms involving $N_{1}$ can be controlled in an elegant way through the introduction of $z_{1}$. Suppose the spurion field $z_{1}$ transforms under the shift symmetry as,

$$
z_{1} \rightarrow \frac{N_{1}}{N_{1}+C} z_{1}
$$

hence $N_{1} z_{1}$ combination remains shift symmetric. At this stage, a discussion on $\mathrm{U}(1)_{R}$ symmetry is pertinent. There exists a global $\mathrm{U}(1)_{R}$ symmetry under which the superpotential $W$ has 2 units of $R$-charges. However note that with the presence of shift symmetric Kähler potential involving $N_{1}, N_{1}$ can not possess a global $\mathrm{U}(1)_{R}$ charge. Therefore $N_{2}$ should carry $R$-charge 2 , while $R$-charges of $N_{1}$ and $z_{1}$ are zero. Furthermore, we consider a $\mathrm{Z}_{2}$ symmetry under which only $N_{1}$ and $N_{2}$ are odd. Combining the shift symmetry, $\mathrm{U}(1)_{R}$ and the $\mathrm{Z}_{2}$ (charges are specified in table 4 ), we can write the general superpotential for $W_{N}$ as

$$
W_{N}^{g}=\left[z_{1} N_{1}+a_{3}\left(z_{1} N_{1}\right)^{3}+\ldots\right] N_{2} .
$$

As the $z_{1}$ gets a vacuum expectation value (vev) $\sim m$ which is small compared to $M_{P}$, we can argue that the shift symmetry is softly broken. Simultaneously the higher order terms (with coefficient $a_{i} \sim \mathcal{O}(1)$ ) are negligibly small and hence we are essentially left with our working superpotential $W_{N}$ in eq. (2.1).

The importance of having this shift symmetry can be understood as discussed below. F-term scalar potential is calculated using the following standard expression,

$$
V_{F}=e^{\frac{K}{M_{P}^{2}}}\left[D_{i} W K_{i j^{*}}^{-1} D_{j^{*}} W^{*}-3 \frac{|W|^{2}}{M_{P}^{2}}\right]
$$

where $D_{i} W=\frac{\partial W}{\partial f_{i}}+K_{i} / M_{P}^{2}$ and the subscript $i$ labels a superfield $f_{i}$. Due to the imposed shift symmetry on $N_{1}$, the Kähler potential (or $e^{K / M_{P}^{2}}$ ) depends only on the imaginary component of $N_{1}$. The real component of $N_{1}$ therefore can be considered to be the inflaton (hereafter denoted by $\chi$ ). Its absence in the Kähler potential allows it to acquire superPlanckian value during inflation, which is a characteristic of large field inflation models. Assuming that during inflation, all other fields (including $N_{2}$ as well) except the inflaton

\footnotetext{
${ }^{1} K_{N}$ also involves $\left|N_{3}\right|^{2}$, which we do not put here for simplifying our discussion.
} 
are stabilized at origin, ${ }^{2}$ the inflationary potential becomes $V_{\chi}=\frac{1}{2} m^{2} \chi^{2}$. The standard slow roll parameters are defined as

$$
\epsilon=\frac{M_{P}^{2}}{2}\left(\frac{V^{\prime}}{V}\right)^{2} ; \eta=\frac{M_{P}^{2} V^{\prime \prime}}{V},
$$

where I denotes the derivative of the potential with respect to inflaton field. Number of $e$-foldings can be calculated by the following,

$$
N_{e}=\frac{1}{M_{P}^{2}} \int_{\chi_{\mathrm{end}}}^{\chi_{*}} \frac{V}{V^{\prime}} d \chi
$$

Other cosmological observables like spectral index $\left(n_{s}\right)$, tensor to scalar ratio $(r)$, curvature perturbation $\operatorname{spectrum}\left(P_{\zeta}\right)$ are given by

$$
n_{s}=1-6 \epsilon+2 \eta ; r=16 \epsilon ; P_{\zeta}=\frac{V}{24 M_{P}^{4} \pi^{2} \epsilon},
$$

respectively. Chaotic inflation with $V_{\chi}$ then predicts

$$
n_{s} \simeq 0.967 \text { and } r \simeq 0.13 \text {, }
$$

where $N_{e}=60$ is considered. Inflation starts at $\chi^{*}=15.5 M_{P}$ and ends at $\chi^{\text {end }}=\sqrt{2} M_{P}$. The value of $m$ turns out to be $\sim 10^{13} \mathrm{GeV}$ so as to produce the correct order of curvature perturbation spectrum $P_{\zeta}=2.2 \times 10^{-9}$. Note that this $m$ falls in the right ballpark for generating light neutrino mass through type-I seesaw. However in view of the recent PLANCK update [2,3], this minimal model is almost outside the $2 \sigma$ region of $n_{s}-r$ plot. So a modification of the minimal model is of utmost importance. As we have mentioned before, there has been some suggestions toward this [24-28]. In this work, our approach to accommodate chaotic inflation within the present experimental limit is to couple it with the supersymmetry breaking sector. This coupling serves as a dynamic modification to the minimal chaotic inflation. To discuss it in detail, in the following section we present a brief summary of the ISS model of dynamical supersymmetry breaking.

\section{SQCD sector and supersymmetry breaking in a metastable vacuum}

It is evident from the F-terms (in particular $F_{N_{2}}=m N_{1}$ ) of $W_{N}$ in eq. (2.1) that during inflation, supersymmetry is broken at a very high scale since the inflaton $(\chi$ field $\equiv$ real part of $N_{1}$ ) takes a non-zero super-Planckian value. However once the inflation is over, the $\chi$ field finally acquires a field-value zero $(\chi=0$ is the global minimum $)$ as evident from the minimization of the potential $V_{\chi}=m^{2} \chi^{2} / 2$. Hence there is no supersymmetry breaking associated with this minimum. It is expected that there should be a small amount of supersymmetry breaking left at the end of inflation so that an effective supersymmetry breaking in the supersymmetric version of the Standard model or its extension can be

\footnotetext{
${ }^{2}$ Particularly for $N_{2}$, this can be ensured by adding a non-canonical term in the Kähler as $\xi\left|N_{2}\right|^{4} /\left[2 M_{P}^{2}\right]$ with $\xi \sim 1[4]$.
} 
introduced. In this work, we consider the inflation sector to be assisted by a separate hidden sector responsible for supersymmetry breaking. ${ }^{3}$ We consider the hidden sector to be described by a supersymmetric gauge theory similar to the one considered in the ISS model of dynamic supersymmetry breaking [30]. Recently a proposal [31, 32] of generating chaotic potential for a strongly interacting supersymmetry gauge theory is analysed which leads to a fractional chaotic inflation. However in our approach we consider the SQCD sector to provide a deformation to the sneutrino contribution to the minimal chaotic inflation, and at the end of inflation, this serves as the hidden sector of the supersymmetry breaking. The effective supersymmetric breaking in the standard supersymmetric gauge and matter sector (MSSM or its extension) requires a mediation mechanism from this hidden sector. Here it is considered to be the gauge mediation.

The ISS model is described by the $\mathcal{N}=1$ supersymmetric $\mathrm{SU}\left(N_{C}\right)$ gauge theory (called the electric theory) with $N_{f}$ flavors of quarks $(Q)$ and antiquarks $(\tilde{Q}) . \Lambda$ is the strong coupling scale of this theory. Below this scale $\Lambda$, the theory is described by its magnetic dual $\mathrm{SU}\left(N=N_{f}-N_{C}\right)$ gauge theory with $N_{f}$ flavors of magnetic quarks $q_{i}^{c}, \tilde{q}_{i}^{c}$ (with $i=1 \ldots N_{f}$ and $c=1 \ldots N$ ). It is interesting to note that this theory is IR free, provided $N_{C}+1 \leq N_{f}<\frac{3}{2} N_{C}$. The elegance of the ISS model lies in its UV completion of the theory. There also exists a $N_{f} \times N_{f}$ gauge singlet meson field $\Phi=Q \tilde{Q} / \Lambda$. With the introduction of quark mass term in the electric theory ( $\mathrm{SU}\left(N_{c}\right)$ gauge theory),

$$
W_{e}=m_{Q} \operatorname{Tr} Q \tilde{Q},
$$

with $m_{Q}<\Lambda$, the IR free magnetic theory becomes

$$
W_{\mathrm{ISS}}=h \operatorname{Tr}(q \Phi \tilde{q})-h \mu^{2} \operatorname{Tr}(\Phi),
$$

along with the dynamical superpotential

$$
W_{\text {dyn }}=N\left(h^{N_{f}} \frac{\operatorname{det} \Phi}{\Lambda^{N_{f}-3 N}}\right)^{\frac{1}{N}} .
$$

where $h \sim \mathcal{O}(1)$ and $\mu \ll \Lambda$ and by duality $\mu^{2}=m_{Q} \Lambda$. Note that there exists a $\mathrm{U}(1)_{R}$ symmetry under which $W_{\text {ISS }}$ and hence $\Phi$ carry $R$-charge of 2-units. R charge of $Q \tilde{Q}$ combination turns out to be two as well from the relation $\Phi=Q \tilde{Q} / \Lambda$. However the $R$ symmetry is explicitly broken by the $W_{\text {dyn }}$ term. All the fields in this sector are considered to be even under the $\mathrm{Z}_{2}$ symmetry considered. The Kähler potential is considered to be canonical in both electric and magnetic theories. It is shown in [17] that there exists a local minimum given by

$$
\langle q\rangle=\left\langle\tilde{q}^{T}\right\rangle=\mu\left(\begin{array}{c}
\mathbb{1}_{N} \\
0_{N_{f}-N}
\end{array}\right),\langle\Phi\rangle=0,
$$

with vacuum energy $V_{\text {ISS }}=N_{c}\left|h^{2} \mu^{4}\right|$. Supersymmetry is broken in this minimum by the rank condition. Note that $W_{\mathrm{dyn}}$ is almost negligible around $\Phi=0$. The interplay between

\footnotetext{
${ }^{3}$ Another approach to accommodate supersymmetry breaking after chaotic inflation is exercised in [29] with an introduction of a Polonyi field.
} 
second term in eq. (3.2) and the $W_{\text {dyn }}$ suggests an existence of a SUSY preserving vacuum at

$$
\langle q\rangle=\left\langle\tilde{q}^{T}\right\rangle=0,\langle\Phi\rangle=\frac{\mu}{h}\left(\epsilon^{\frac{N_{f}-3 N}{N_{c}}}\right)^{-1} \mathbb{1}_{N_{f}},
$$

where $\epsilon=\frac{\mu}{\Lambda}$ and the corresponding vacuum energy $V_{0}=0$. With $\epsilon \ll 1$, it was shown in [17] that the local minima in eq. (3.4) is a metastable one.

\section{Interaction between neutrino and SQCD sectors}

We consider $W_{N}$ as the superpotential describing the inflation with $N_{1}$ playing the role of inflaton. In this section our endeavor is to couple the inflaton with the SQCD sector. We assume that the two sectors can communicate with each other only through gravity. The lowest dimensional operator consistent with the set-up is therefore given by,

$$
W_{\text {Int }}=\beta \frac{N_{1}^{2} \operatorname{Tr}(Q \tilde{Q})}{M_{P}},
$$

where $\beta$ is a coupling constant. We consider $\beta$ to be much less than unity. Similar to $W_{\text {ISS }}, W_{\text {Int }}$ also respects the $\mathrm{U}(1)_{R}$ and hence linear in $\operatorname{Tr}(Q \tilde{Q})$ having $R$-charge 2 . Among $N_{1}$ and $N_{2}$, it is therefore the $N_{1}$ field only which can couple $\left(N_{2}\right.$ carries 2 units of $R$ charge) with the ISS sector. Since the interaction between the two sectors are assumed to be mediated by gravity only, the interaction term is expected to be $M_{P}$ suppressed. Hence $W_{\text {Int }}$ in eq. (4.1) serves as the minimal description of the interaction between the two sectors. Being a shift-symmetry breaking parameter, the origin of $\beta$ can be explained with the introduction of another spurion field $z_{2}$ which transforms as $z_{2} \rightarrow \frac{N_{1}^{2}}{\left(N_{1}+C\right)^{2}} z_{2}$ under shift symmetry. We consider $z_{2}$ to be even under the $\mathrm{Z}_{2}$ symmetry and it does not carry any $R$ charge. On the other hand, $Q \tilde{Q}$ combination is even under $\mathrm{Z}_{2}$. We introduce another discrete symmetry $\mathrm{Z}_{4}$ under which $z_{2}$ carries a charge $i$ as well as $Q \tilde{Q}$ carrying charge $-i$. Hence $m_{Q}$ also carries a $\mathrm{Z}_{4}$ charge $i$ as seen from eq. (3.1). Application of this symmetry forbids dangerous term like $\frac{\left(z_{2} N_{1}^{2}\right) z_{1} N_{1} N_{2}}{M_{P}^{3}}$. Hence a general superpotential involving $z_{2} N_{1}^{2}$ can be obtained as

$$
W_{\text {Int }}^{g}=\frac{\operatorname{Tr}(Q \tilde{Q})}{M_{P}}\left[\frac{z_{2} N_{1}^{2}}{M_{P}}+b_{5} \frac{\left(z_{2} N_{1}^{2}\right)^{5}}{M_{P}^{13}}+\ldots \ldots\right]
$$

where $b_{5}$ corresponds to respective coupling. Terms involving quadratic, cubic, and quartic powers of $\left(z_{2} N_{1}^{2}\right)$ are not allowed from the $\mathrm{Z}_{4}$ charge assignment as considered. ${ }^{4}$ Therefore $\beta$ is obtained through $\beta=\left\langle z_{2}\right\rangle / M_{P}$. Note that with $\beta \sim 10^{-3}$ (as we will see), terms with $b_{5}$ and higher orders are negligibly small.

Note that this interaction term in addition to the quark mass term $m_{Q}$ present in $W_{e}$ (see eq. (3.1)), generates an effective mass for the electric quarks, $m_{Q}^{\prime}=\beta \frac{N_{1}^{2}}{M_{P}}+m_{Q}$. Here we are particularly interested in the case when the effective mass of the quarks, $m_{Q}^{\prime}$, becomes larger than the cut-off scale $\Lambda$, i.e. when $m_{Q}^{\prime} \gg \Lambda$. Since $m_{Q}$ is considered to

\footnotetext{
${ }^{4}$ With this new $\mathrm{Z}_{4}$ symmetry, term like $\frac{\left(z_{2} N_{1}^{2}\right) z_{1} N_{1} N_{2}}{M_{P}^{3}}$ will be allowed in $W_{\text {Int }}$. However contribution of this term will be negligibly small.
} 
be less than $\Lambda$ in the ISS set-up, this situation can be achieved when the inflaton field $N_{1}$ satisfies, $N_{1} \gg\left[\Lambda M_{P} / \beta\right]^{1 / 2}$.

These heavy quarks can then be integrated out [30] to form an effective theory with a field dependent dynamical scale, $\Lambda_{\text {eff }}\left(N_{1}\right)$. As all the quarks are getting large masses, the effective theory becomes a pure gauge theory with no flavors. $\Lambda_{\text {eff }}$, can be determined by the standard scale matching of the gauge couplings of two theories at an energy scale $E=m_{Q}^{\prime}$. With $g_{N_{c}, N_{f}}$ and $g_{N_{c}, 0}$ are the gauge couplings of the $\mathrm{SU}\left(N_{c}\right)$ gauge theory with $N_{f}$ flavors of quarks $(Q, \tilde{Q})\left(E>m_{Q}^{\prime}\right)$ and pure gauge theory with $N_{f}=0\left(E<m_{Q}^{\prime}\right)$ respectively, the condition $g_{N_{c}, N_{f}}\left(m_{Q}^{\prime}\right)=g_{N_{c}, 0}\left(m_{Q}^{\prime}\right)$ gives

$$
\left(\frac{m_{Q}^{\prime}}{\Lambda}\right)^{b}=\left(\frac{m_{Q}^{\prime}}{\Lambda_{\mathrm{eff}}}\right)^{b_{\mathrm{eff}}}
$$

where $b=3 N_{c}-N_{f}$ and $b_{\text {eff }}=3 N_{c}$ are the respective beta functions of gauge couplings of the two theories. $\Lambda_{\text {eff }}$ in our set up turns out to be

$$
\Lambda_{\mathrm{eff}} \simeq\left(\frac{\beta N_{1}^{2}}{M_{P}}\right)^{(1-p)} \Lambda^{p}
$$

where $p=\frac{b}{b_{\text {eff }}}$ and $m_{Q}^{\prime}$ is mostly dominated by $\frac{\beta N_{1}^{2}}{M_{P}}$ term (i.e. when $N_{1} \gg\left[\Lambda M_{P} / \beta\right]^{1 / 2}$ and $m_{Q}$ being much smaller than $\Lambda$ can be neglected). As all the flavors are integrated out, the superpotential describing the effective theory is generated via gaugino condensation and is given by [30]

$$
W_{\text {Int }}^{\text {eff }}=N_{c} \Lambda_{\text {eff }}^{3}=N_{c}\left(\beta \frac{N_{1}^{2}}{M_{P}}\right)^{3(1-p)} \Lambda^{3 p} .
$$

Below we study the impact of this term on inflation governed by $W_{N}$.

\section{Modified chaotic potential and its implications to inflationary dynam- ics}

Here we will study the inflationary dynamics based on the superpotential,

$$
W_{\mathrm{Inf}}=W_{N}+W_{\mathrm{Int}}^{\mathrm{eff}}=m N_{1} N_{2}+N_{c}\left(\frac{\beta N_{1}^{2}}{M_{P}}\right)^{3(1-p)} \Lambda^{3 p},
$$

when $N_{1} \gg\left[\Lambda M_{P} / \beta\right]^{1 / 2}$. Note that it indicates a modification of the chaotic inflationary potential $V_{\chi}$ obtained from $W_{N}$ only. In this section, we will study the outcome of this modified superpotential in terms of prediction of parameters involved in inflation. Depending upon $p$, the superpotential may contain fractional powers of $N_{1}$. In ref. [33], superpotential with non-integer power of superfields has been studied. It is shown there that the form of Kähler potential remains same irrespective of integer or non-integer power of superfields involved in the superpotential.

The Kähler potential is considered to be the same as $K_{N}$ in eq. (2.2). We can write

$$
N_{1}=\frac{\chi+i \eta}{\sqrt{2}} \text { and } N_{2}=\frac{\sigma+i \delta}{\sqrt{2}}
$$


As discussed in section 2, we choose $\chi$, the real component of $N_{1}$, as inflaton. Using eq. (2.2), (2.5) and eq. (5.1), the scalar potential involving $\chi$ and $\sigma$ is given by

$$
\begin{aligned}
V_{\operatorname{Inf}}(\tilde{\chi}, \tilde{\sigma})=M_{P}^{4} e^{\tilde{\sigma}^{2} / 2}[ & \frac{\tilde{m}^{2} \tilde{\sigma}^{2}}{2}+\frac{\tilde{m}^{2} \tilde{\chi}^{2}}{2}\left(1-\frac{\tilde{\sigma}^{2}}{2}+\frac{\tilde{\sigma}^{4}}{4}\right) \\
& +3(1-p) A \tilde{\chi}^{5-6 p} \tilde{\sigma}+A \tilde{\chi}^{7-6 p}\left(-\frac{\tilde{\sigma}}{2}+\frac{\tilde{\sigma}^{3}}{8}\right) \\
& \left.+\frac{9}{2 \tilde{m}^{2}}(1-p)^{2} A^{2} \tilde{\chi}^{10-12 p}+\frac{A^{2}}{2 \tilde{m}^{2}} \tilde{\chi}^{12-12 p}\left(-\frac{3}{8}+\frac{\tilde{\sigma}^{2}}{16}\right)\right],
\end{aligned}
$$

where $A=\frac{\beta^{3(1-p)} \tilde{\tilde{N}} N_{c} \tilde{\Lambda}^{3 p}}{2^{1-3 p}}$ and tilde indicates that the corresponding variable or parameter is scaled in terms of $M_{P}$, e.g. $\tilde{\sigma}=\frac{\sigma}{M_{P}}$. We follow this notation throughout this section only. As the electric quarks degrees of freedom $(Q, \tilde{Q})$ are integrated out we will not consider quarks anymore, as long as $N_{1} \gg \sqrt{\frac{\Lambda M_{P}}{\beta}}$. Now one can wonder what happened to other two fields $\eta$ and $\delta$. Due to the presence of $e^{K}$ factor in the scalar potential $V_{F}$ the effective mass of $\eta$ during inflation will be large compared to inflaton mass $\left(m_{\eta}^{2} \sim 6 H_{\mathrm{Inf}}^{2}+m^{2}\right)$ and hence it will quickly settle down to origin. We have checked numerically that the other field $\delta$ also settles at origin during inflation, having mass more than the Hubble.

In this case, dynamics of inflation belongs to $\tilde{\chi}-\tilde{\sigma}$ plane. Note that in case of standard chaotic inflation as discussed in section 2 , the $\tilde{\sigma}$ field is considered to be at origin during inflation. Contrary to that, the dynamic modification of the scalar potential governed by $W_{\text {Int }}^{\text {eff }}$ forces the $\tilde{\sigma}$ field to have a nonzero vacuum expectation value in our case. Similar type of scenarios are discussed in $[16,24]$. In order to get $\langle\tilde{\sigma}\rangle$ in terms of $\tilde{\chi}$, derivative of the scalar potential with respect to $\tilde{\sigma}$ and yields

$$
\begin{aligned}
\frac{\partial V_{\text {Inf }}}{\partial \tilde{\sigma}}=e^{\frac{\tilde{\sigma}^{2}}{2}}[- & \frac{A \tilde{\chi}^{5-6 p}}{2}\left(\tilde{\chi}^{2}-6+6 p\right)+\tilde{\sigma}\left\{\tilde{m}^{2}+\tilde{\chi}^{10-12 p} \frac{A^{2}}{2 \tilde{m}^{2}}\left(9-18 p+9 p^{2}-\frac{\tilde{\chi}^{2}}{4}\right)\right\} \\
& +\tilde{\sigma}^{2}\left\{A \tilde{\chi}^{5-6 p}\left(3-3 p-\frac{\tilde{\chi}^{2}}{8}\right)\right\}+\tilde{\sigma}^{3}\left\{\frac{\tilde{m}^{2}}{2}\left(1+\frac{\tilde{\chi}^{2}}{2}\right)+\frac{\tilde{\chi}^{12-12 p}}{32} \frac{A^{2}}{\tilde{m}^{2}}\right\} \\
& \left.+\tilde{\sigma}^{4}\left\{\tilde{\chi}^{7-6 p} \frac{A}{8}\right\}+\tilde{\sigma}^{5}\left\{\frac{\tilde{\chi}^{2} \tilde{m}^{2}}{8}\right\}\right] .
\end{aligned}
$$

In order to minimize the scalar potential, we equate the above expression to zero (i.e.

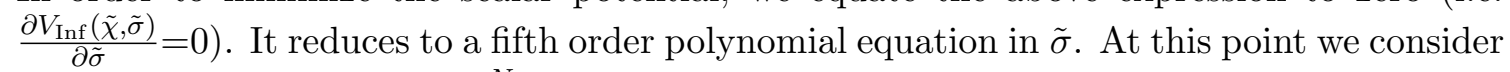
a specific value of $p\left(=1-\frac{N_{f}}{3 N_{c}}\right)$, the choice of which is guided by the construction of the ISS framework and a possible realization of $\mathrm{U}(1)_{R}$ breaking through baryon deformation as we will discuss in section 8. Comparing the relative magnitudes of the terms involved in the fifth order polynomial and considering $\tilde{\sigma}$ to be sub-Planckian, we solve the equation for $\tilde{\sigma}$ in a perturbative way, the details of which is given in appendix A. Once $\langle\tilde{\sigma}\rangle$ is obtained in terms of $\tilde{\chi}$, we replace $\tilde{\sigma}$ by its VEV in eq. (5.3) and potential responsible for inflation now becomes function of $\tilde{\chi}$ only. Due to its very complicated functional dependence on $\tilde{\chi}$, we have not presented $V_{\text {Inf }}$ here. Instead in figure 1 we have depicted the potential $V(\tilde{\chi})$ in terms of $\tilde{\chi}$ for $p=4 / 7$ (indicated by dashed line). Note that this potential is indeed flatter compared to the standard sneutrino chaotic inflation potential [15], indicated in figure 1 by the solid line. 


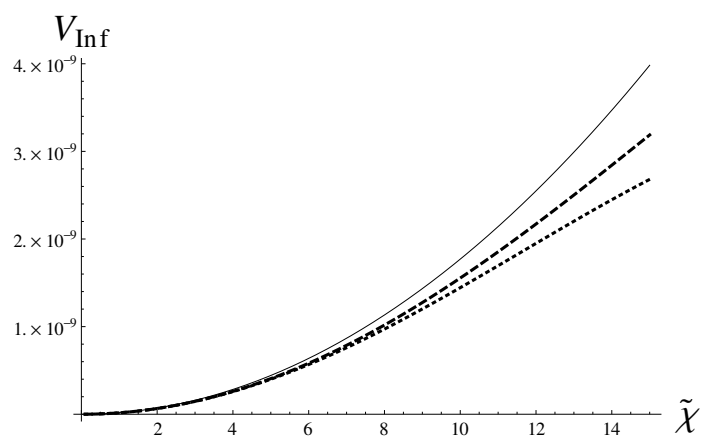

Figure 1. Examples of inflation potential $\left(V_{\text {Inf }}\right)$ against $\tilde{\chi}$ are presented. The solid, large-dashed and small-dashed curves represent (I) minimal chaotic potential with $V_{\operatorname{Inf}}(\tilde{\chi})=\frac{1}{2} \tilde{m}^{2} \tilde{\chi}^{2}$, (II) modified $V_{\text {Inf }}(\tilde{\chi})$ obtained from our set-up with $\alpha=0$ and (III) modified $V_{\text {Inf }}(\tilde{\chi})$ with $\alpha=7 \times 10^{-4}$ respectively.

For completeness a shift symmetry breaking parameter $(\alpha)$ in the Kähler potential can also be introduced. The modified Kähler potential will look like as

$$
K=K_{N}-\alpha \frac{\left(N_{1}+N_{1}^{\dagger}\right)^{2}}{2}
$$

with $\alpha \ll 1$. The scalar potential in eq. (5.3) will be modified and takes a further complicated form. In this case we obtain the scalar potential as a function of $\tilde{\chi}$ in a similar way. In figure (1) we also plot $V_{\operatorname{Inf}}(\tilde{\chi})$ including the nonzero value of $\alpha\left\{\sim 7 \times 10^{-4}\right\}$ represented by dashed line. It is to be noted that introduction of $\alpha$ makes the shape of $V_{\operatorname{Inf}}(\tilde{\chi})$ even flatter.

\section{$6 \quad$ Results}

End of inflation occurs when slow roll parameters become unity i.e. $\epsilon, \eta \simeq 1$. Solving the equalities we find inflaton field value at the end of inflation $\chi_{\text {end }} \simeq \sqrt{2} M_{P}$. Now it is visible from $V_{\operatorname{Inf}}(\chi, \sigma)$ and eq. (5.4) that we are left with two free parameters $m$ and $\Lambda$ once $p$ is fixed. The value of $\beta$ is taken to be $\mathcal{O}\left(10^{-3}\right)$ so that it satisfies $\Lambda<m_{Q}^{\prime}<M_{P}$. We have performed a scan over these parameters and few of our findings are tabulated in table 1 . We find $m$ is mostly restricted by the value of curvature perturbation, while $\Lambda$ helps decreasing $r$. We consider $m$ to be below $\Lambda$. Also, we consider effects of non-zero $\alpha$ which is provided in table 2 .

We find from table 1 that corresponding to $\Lambda=1.05 \times 10^{-3} M_{P}$, values of $r \sim 0.079$ and $n_{s} \sim 0.96$ can be achieved with $m \sim 5.5 \times 10^{-6} M_{P}$. To compare, with the same $\Lambda$, a somewhat lower value of $r \sim 0.069$ and $n_{s} \sim 0.96$ are obtained with $m=5.5 \times 10^{-6} M_{P}$ and $\alpha=0.0003$. In obtaining table 2 , we have kept $\frac{\alpha \chi^{2}}{M_{P}^{2}} \ll 1$. In figure 2 , we indicate the respective points of table 1 by black dots and note that those points fall within the $2 \sigma$ allowed range of $n_{s}-r$ plot from PLANCK $2015[2,3]$ safely. The solid line for $N_{e}=60$ indicates the possible set of points (including the ones from table 1) that describe $n_{s}$ and $r$ for different values of $\Lambda$. Similarly the other solid line corresponds to the set of points 


\begin{tabular}{|l|l|l|l|l|}
\hline$\Lambda$ & $m$ & $\chi^{*}$ & $r$ & $n_{s}$ \\
\hline $8.90 \times 10^{-4}$ & $5.75 \times 10^{-6}$ & 14.95 & 0.099 & 0.965 \\
\hline $1.05 \times 10^{-3}$ & $5.47 \times 10^{-6}$ & 14.55 & 0.079 & 0.961 \\
\hline $1.18 \times 10^{-3}$ & $4.91 \times 10^{-6}$ & 13.92 & 0.052 & 0.954 \\
\hline
\end{tabular}

Table 1. Predictions for $r, n_{s}$ and $\chi^{*}$ are provided for sets of values of parameters $m, \Lambda$ involved in $V_{\text {Inf }}$. The dataset corresponds to $N_{e}=60, \alpha=0, p=4 / 7, \beta=1.5 \times 10^{-3}$ and values of $(m, \Lambda$, $\chi^{*}$ ) are in $M_{P}$ unit.

\begin{tabular}{|l|l|l|l|l|}
\hline$\alpha$ & $m$ & $\chi^{*}$ & $r$ & $n_{s}$ \\
\hline 0.0003 & $5.390 \times 10^{-6}$ & 14.271 & 0.069 & 0.960 \\
\hline 0.0005 & $5.300 \times 10^{-6}$ & 14.067 & 0.063 & 0.959 \\
\hline 0.0007 & $5.156 \times 10^{-6}$ & 13.841 & 0.055 & 0.957 \\
\hline
\end{tabular}

Table 2. Predictions for $r, n_{s}$ and $\chi^{*}$ are provided for sets of values of parameters $m$ and $\Lambda$ involved in $V_{\text {Inf. }}$. The dataset corresponds to $N_{e}=60, \Lambda=1.05 \times 10^{-3} M_{P}, p=4 / 7, \beta=1.5 \times 10^{-3}$ and values of $\left(m, \chi^{*}\right)$ are in $M_{P}$ unit.

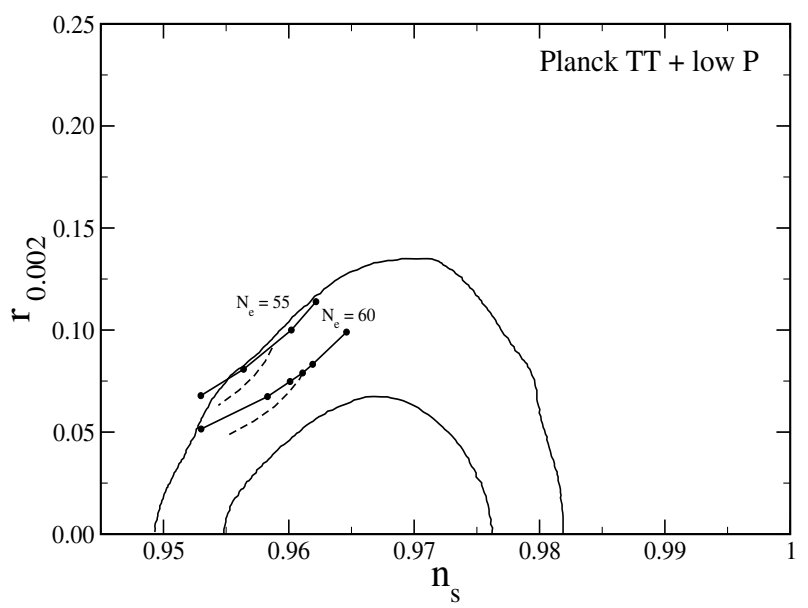

Figure 2. Predictions for $n_{s}$ and $r$ as obtained including dataset from the modified chaotic inflation model (from table 1) indicated by dark dots for $N_{e}=60$. A solid line joining them represents the prediction of $n_{s}$ and $r$ while $\Lambda$ is varied. Similar predictions for $N_{e}=55$ are also included. The dashed lines correspond to the predictions by varying $\alpha$ while value of $\Lambda$ is fixed at $1.1 \times 10^{-3} M_{P}$ (for $\left.N_{e}=55\right)$ and $1.05 \times 10^{-3} M_{P}\left(\right.$ for $\left.N_{e}=60\right)$.

for $N_{e}=55$. The dashed lines describe the effect of introducing $\alpha$. Now we can have an estimate of the mass of the $\delta$ field $\left(m_{\delta}\right)$ during inflation. For $\Lambda=1.05 \times 10^{-3} M_{P}$, $m=5.5 \times 10^{-6} M_{P}$ we found numerically $\frac{m_{\delta}}{H_{\text {Inf }}}=\sim 1.2$ during inflation. This ensures $\delta$ field to be stabilized at origin. On the other hand, $\frac{m_{\sigma}}{H_{\text {Inf }}}$ is found to be $\sim 2.5$ which indicates that the fluctuation of $\sigma$-field about $\langle\sigma\rangle$ (in terms of $\chi$ ) is almost negligible. 


\section{Dynamics after inflation}

Once the inflation is over, the field $\chi$ rolls down along the path as shown in figure 1 and $\langle\sigma\rangle$ also follows its VEV which is $\chi$ dependent. Note that at the end of inflation, $N_{1}$ still satisfies $N_{1} \gg \sqrt{\frac{\Lambda M_{P}}{\beta}}$ condition. However once $N_{1}<\Lambda$ is realized, we need to relook into the term responsible for dynamic modification of chaotic inflation. As in this situation $m_{Q}^{\prime} \ll \Lambda$, the electric quarks $(Q, \tilde{Q})$ can not be integrated out anymore and we can use the magnetic dual description of the ISS sector similar to eq. (3.2) and (3.3). Therefore the superpotential for the ISS, describing the magnetic dual theory and the RH neutrino becomes

$$
W_{m}=h \operatorname{Tr}(q \Phi \tilde{q})-h \mu^{2} \operatorname{Tr} \Phi-\frac{\beta N_{1}^{2} \operatorname{Tr}(\Phi) \Lambda}{M_{P}}+m N_{1} N_{2} .
$$

To discuss what happens to the $N_{1}$ and the fields involved in SUSY breaking sector, let us calculate the F-terms as follows

$$
\begin{aligned}
F_{\Phi_{i j}} & =h \tilde{q}_{c}^{i} q_{j}^{c}-\left(h \mu^{2}+\frac{\beta \Lambda N_{1}^{2}}{M_{P}}\right) \delta_{i j}, \\
F_{q_{i}} & =h \Phi_{j}^{i} \tilde{q}^{j} ; F_{\tilde{q}^{j}}=h q_{i} \Phi_{j}^{i}, \\
F_{N_{1}} & =-\frac{2 \beta \Lambda}{M_{P}} N_{1} \operatorname{Tr}(\Phi)+m N_{2}, \\
F_{N_{2}} & =m N_{1} .
\end{aligned}
$$

Similar to the original ISS model, here also all the $F$-terms can not be set to zero simultaneously and hence the supersymmetry breaking is realized. The scalar potential becomes

$$
\begin{aligned}
V= & \left(N_{f}-N_{c}\right)\left|h q \tilde{q}-h \mu^{2}-\frac{\beta N_{1}^{2} \Lambda}{M_{P}}\right|^{2}+N_{c}\left|h \mu^{2}+\frac{\beta N_{1}^{2} \Lambda}{M_{P}}\right|^{2} \\
& +|h q \Phi|^{2}+|h \Phi \tilde{q}|^{2}+\left|m N_{2}-\frac{2 \beta N_{1} \operatorname{Tr}(\Phi) \Lambda}{M_{P}}\right|^{2}+m^{2}\left|N_{1}\right|^{2} .
\end{aligned}
$$

Supergravity corrections are not included in this potential as below the scale $\Lambda$, the SUGRA corrections become negligible. As long as $N_{1}$ remains nonzero, the minimum of $q, \tilde{q}, \Phi$ and $N_{2}$ are given by

$$
\langle q\rangle=\langle\tilde{q}\rangle=\sqrt{\mu^{2}+\frac{\beta \Lambda N_{1}^{2}}{h M_{P}}}\left(\begin{array}{l}
\mathbb{1}_{N_{f}-N_{c}} \\
0_{N_{c}}
\end{array}\right),\langle\Phi\rangle=0,\left\langle N_{2}\right\rangle=0 .
$$

A point related to $\langle\Phi\rangle$ is pertinent here. In the ISS set-up, a classical flat direction is present in a smaller subspace of $\Phi$ which is essentially lifted by the Coleman-Weinberg (CW) [17] correction and $\langle\Phi\rangle=0$ is achieved. In our set-up there exists a supergravity influenced mass $\sim \frac{m^{2} N_{1}^{2}}{M_{P}^{2}}$ for all the components of $\Phi$ once a canonical Kähler potential is assumed. This helps $\Phi$ to settle at origin. However once $N_{1}$ moves to its own minimum which is at $N_{1}=0$, this induced mass term vanishes and at that stage, CW correction 
becomes important to lift the flatness. For our purpose, we consider $\langle\Phi\rangle$ to be at zero which serves as the local minimum of the theory.

We will now concentrate on the potential involving $N_{1}$. Assuming all other fields are stabilized at their $\mathrm{VEV}$ (with $\left\langle N_{2}\right\rangle=0$ as $\langle\Phi\rangle=0$ ) the scalar potential involving $N_{1}$ becomes

$$
V_{N_{1}}=N_{c}\left|h \mu^{2}+\frac{\beta N_{1}^{2} \Lambda}{M_{P}}\right|^{2}+m^{2}\left|N_{1}\right|^{2} .
$$

Splitting $N_{1}$ into real and imaginary components we get

$$
\begin{aligned}
V_{N_{1}}(\chi, \eta)= & N_{c} h^{2}|\mu|^{4}+\left(\chi^{4}+\eta^{4}+2 \chi^{2} \eta^{2}\right) \frac{N_{c} \Lambda^{2} \beta^{2}}{4 M_{P}^{2}} \\
& +\eta^{2}\left(\frac{m^{2}}{2}-\frac{h N_{c} \beta \mu^{2} \Lambda}{M_{P}}\right)+\chi^{2}\left(\frac{m^{2}}{2}+\frac{h N_{c} \beta \mu^{2} \Lambda}{M_{P}}\right) .
\end{aligned}
$$

By equating $\frac{\partial V(\chi, \eta)}{\partial \eta}$ with zero, we find $\langle\eta\rangle=0$ provided $m^{2}>\frac{2 h N_{c} \beta \mu^{2} \Lambda}{M_{P}}$. This condition is easily satisfied in our analysis for the allowed range of $m, \Lambda$ and $N_{c}$ with the observation that $\mu$ can be at most $\sim 10^{12} \mathrm{GeV}$ for gravity mediated supersymmetry breaking and $h \sim \mathcal{O}(1)$. In case of gauge mediatioin $\mu$ can be even smaller. Therefore setting $\eta=0$ eq. (7.9) becomes

$$
V_{\chi}=N_{c} h^{2}|\mu|^{4}+\chi^{4} \frac{N_{c} \beta^{2} \Lambda^{2}}{4 M_{P}^{2}}+\chi^{2}\left(\frac{m^{2}}{2}+\frac{h N_{c} \beta \mu^{2} \Lambda}{M_{P}}\right) .
$$

It clearly shows that $\chi=0$ is the minimum of the potential with the vacuum energy $V_{0}=N_{c} h^{2}|\mu|^{4}$. So when $N_{1}$ settles to zero and reheats, the SQCD sector is essentially decoupled as $W_{\text {Int }}$ vanishes with $N_{1}=0$. At this stage the ISS sector stands for the supersymmetry breaking in the metastable minima described by eq. (3.4) and $\langle\chi\rangle=0$. Reheat will depend on the coupling of $N_{1}$ with other SM fields.

\section{Dynamical breaking of $\mathrm{U}(1)_{R}$}

In the construction of the ISS picture of realizing supersymmetry breaking dynamically, $\mathrm{U}(1)_{R}$ symmetry plays an important role. The superpotential $W$ carries $R$-charge of two units. The $\Phi$ field being linear in the superpotential must also carry the $R$-charge 2 and it is not broken as $\langle\Phi\rangle=0$. A lot of exercises have been performed to achieve $R$ symmetry breaking in order to give mass to the gauginos. One such interesting approach is through the baryon deformation of $W_{m}$ suggested by [22]. In [22] the authors considered the superpotential (for the magnetic theory)

$$
W=\Phi_{i j} q_{i} \tilde{q}_{j}-\mu^{2} \Phi_{i j}+m_{q} \epsilon_{a}^{r} \epsilon_{b}^{s} q_{r}^{a} q_{s}^{b},
$$

with $N_{f}=7$ and $N_{c}=5$, where $r, s=1,2$ and $i, j=1, \ldots, 7$ and $\mu^{2}=m_{Q} \Lambda$. R-charges of $q, \tilde{q}$ and $\Phi$ are provided in table 3 and reason behind this choice is elaborated in appendix B. With the specific choice of $N_{f}$ and $N_{c}$, the last term is a singlet under the gauge group in the magnetic theory. It represents the baryon deformation, introduction of which shifts 


\begin{tabular}{|l|l|l|l|l|}
\hline Fields & $q$ & $\tilde{q}$ & $\Phi$ & $m_{Q}$ \\
\hline $\mathrm{U}(1)_{R}$ & 1 & -1 & 2 & 0 \\
\hline $\mathrm{Z}_{2}$ & 1 & 1 & 1 & 1 \\
\hline $\mathrm{Z}_{4}$ & 1 & $i$ & $-i$ & $i$ \\
\hline $\mathrm{Z}_{4}^{\prime}$ & 1 & 1 & 1 & 1 \\
\hline
\end{tabular}

Table 3. $\mathrm{U}(1)_{R}, \mathrm{Z}_{2}, \mathrm{Z}_{4}$ and $\mathrm{Z}_{4}^{\prime}$ charges of various fields involved in the modified ISS model.

the $\langle\Phi\rangle$ to a nonzero value $\sim m_{q}$ and thereby breaking $R$-symmetry spontaneously. In realizing this set-up it was assumed the associated global symmetry $\mathrm{SU}\left(N_{f}=7\right)$ is broken down to $\mathrm{SU}(5) \times \mathrm{SU}(2)$ and the $\mathrm{SU}(5)$ after gauging can therefore be identified with the parent of the Standard Model gauge group. We follow this suggestion for breaking the $\mathrm{U}(1)_{R}$ and argue that this approach and the conclusion of [22] are effectively unaltered by the additional interaction between the SQCD-sector and the inflation sector. In view of eq. (8.1), the charges of $q, \tilde{q}$ and $\Phi$ under the discrete symmetries introduced in our framework are provided in table 3 .

With the introduction of the additional interaction term $\left(W_{\text {Int }}\right)$, we can define an effective $\mu_{\text {eff }}$ in the superpotential with $\mu_{\text {eff }}^{2}=\mu^{2}+\Lambda \frac{N_{1}^{2}}{M_{P}}$. We find the minimal choice as in [22] $N_{f}=7$ and $N_{c}=5$, does not provide enough modification (or flatness) in terms of the inflaton potential. So we have chosen $N_{f}=9$ and $N_{c}=7$ so that the gauge group in the magnetic theory remains $\mathrm{SU}(2)$ as in [22]. The global symmetry $\mathrm{SU}(9)$ is expected to be broken into $\mathrm{SU}(2) \times \mathrm{SU}(7)$ explicitly. Taking both these modifications into account, we expect the conclusions of [22] are essentially remain unchanged, i.e. $\langle\Phi\rangle$ is shifted by an amount $\sim m_{q} \sim \mathcal{O}(\mu)$ and hence gauginos become massive. The detailed discussion of the $\mathrm{U}(1)_{R}$ breaking is beyond the scope of this paper. Note that this sort of mechanism for breaking $\mathrm{U}(1)_{R}$ holds for $\mu \geq 10^{5} \mathrm{TeV}$ as found in [22]. The upper limit on $\mu$ can be $\sim 10^{12} \mathrm{GeV}$, where gravity mediation dominates over gauge mediation. This range of $\mu$ is consistent in satisfying $m^{2}>\frac{2 N_{c} \beta \mu^{2} \Lambda}{M_{P}}$ relation also which keeps the $\langle\eta\rangle$ at origin as discussed in section 7 .

\section{$9 \quad$ Neutrino masses and mixing}

We will discuss reheating and generation of light neutrino masses through the superpotential

$$
W=W_{m}+m_{3} N_{3}^{2}+h_{i \alpha} N_{i} L_{\alpha} H_{u}
$$

$W_{m}$ is as described in eq. (7.1). The second and third terms represent the mass term for the third RH neutrino and the neutrino Yukawa couplings with all three RH neutrinos respectively. Note that the superpotential respects the $\mathrm{U}(1)_{R}$ symmetry and therefore the choice of $R$-charges of the $\mathrm{SU}(2)_{L}$ lepton doublets further restricts the Yukawa interaction terms. 


\begin{tabular}{|l|l|l|l|l|l|l|l|l|l|l|}
\hline Fields & $N_{1}$ & $N_{2}$ & $N_{3}$ & $L_{1}$ & $L_{2}$ & $L_{3}$ & $H_{u, d}$ & $z_{1}$ & $z_{2}$ & $z_{3}$ \\
\hline $\mathrm{U}(1)_{R}$ & 0 & 2 & 1 & 2 & 0 & 0 & 0 & 0 & 0 & 0 \\
\hline $\mathrm{Z}_{2}$ & -1 & -1 & 1 & 1 & -1 & -1 & 1 & 1 & 1 & -1 \\
\hline $\mathrm{Z}_{4}$ & 1 & 1 & 1 & 1 & 1 & 1 & 1 & 1 & $i$ & 1 \\
\hline $\mathrm{Z}_{4}^{\prime}$ & 1 & 1 & 1 & $-i$ & 1 & 1 & 1 & 1 & 1 & $i$ \\
\hline
\end{tabular}

Table 4. $\mathrm{U}(1)_{R}, \mathrm{Z}_{2}, \mathrm{Z}_{4}$ and $\mathrm{Z}_{4}^{\prime}$ charges of the RH neutrinos, Higgs and Lepton doublets.

With one such typical choice of $R$-charges (only) specified in table-4, the allowed Yukawa terms are given by,

$$
W_{Y} \supset h_{11} N_{1} L_{1} H_{u}+h_{22} N_{2} L_{2} H_{u}+h_{23} N_{2} L_{3} H_{u} .
$$

The coefficient $h_{11}$ can be explained through the vev of another spurion $z_{3}$ which transforms similar to $z_{1}$ under shift symmetry while odd under the $\mathrm{Z}_{2}$ symmetry considered. A term in the superpotential $\left(y_{1} z_{3} N_{1} L_{1} H_{u}\right) / M_{P}$ then generates $h_{11}=y_{1}\left\langle z_{3}\right\rangle / M_{P}$. Here we incorporate another discrete symmetry $\mathrm{Z}_{4}^{\prime}$ under which $z_{3}$ has charge $i$ and $L_{1}$ carries $-i$. All the other fields transform trivially under $\mathrm{Z}_{4}^{\prime}$ as seen from table 4 . The new $\mathrm{Z}_{4}^{\prime}$ helps disallow the unwanted terms ${ }^{5}$ like $\frac{\left(z_{3} N_{1}\right) z_{1} N_{1} N_{2}}{M_{P}^{2}}$ and $\frac{z_{3} N_{1} \operatorname{Tr} Q \tilde{Q}}{M_{P}}$.

The superpotential in eq. (7.1) and eq. (9.1) and $W_{Y}$ in eq. (9.2) determine the structure of the RH neutrino mass matrix and the Dirac neutrino mass matrix as

$$
M_{R}=\left(\begin{array}{ccc}
\varepsilon_{m} & m & 0 \\
m & 0 & 0 \\
0 & 0 & m_{3}
\end{array}\right) ; m_{D}=\left\langle H_{u}\right\rangle\left(\begin{array}{ccc}
h_{11} & 0 & 0 \\
0 & h_{22} & h_{23} \\
0 & 0 & 0
\end{array}\right),
$$

with $\varepsilon_{m}=\frac{\beta\langle\Phi\rangle \Lambda}{M_{P}} \ll m$. Here we have incorporated the $\langle\Phi\rangle$ related to the deformation as discussed in section 8. Light neutrino mass-matrix can therefore be obtained from the type-I seesaw [34] contribution $m_{\nu}=m_{D}^{T} \frac{1}{M_{R}} m_{D}$ and is given by

$$
m_{\nu}=\frac{\left\langle H_{u}\right\rangle^{2}}{m}\left(\begin{array}{ccc}
0 & h_{11} h_{22} & h_{11} h_{23} \\
h_{11} h_{22} & -\frac{\varepsilon_{m} h_{22}^{2}}{m} & -\frac{\varepsilon_{m} h_{22} h_{23}}{m} \\
h_{11} h_{23} & -\frac{\varepsilon_{m} h_{22} h_{23}}{m} & -\frac{\varepsilon_{m} h_{22}^{2}}{m}
\end{array}\right) .
$$

Note that all the terms involving $\varepsilon_{m} / m$ are much smaller compared to the 12(21) and 13(31) entries of $m_{\nu}$. Once the terms proportional to $\varepsilon_{m} / m$ are set to zero, $m_{\nu}$ coincides with the neutrino mass matrix proposed in [35] leading to an inverted hierarchical spectrum of light neutrinos. The above texture of $m_{\nu}$ in eq. (9.4) then predicts

$$
\begin{aligned}
m_{\nu_{1}} & \simeq m_{\nu_{2}} \simeq \sqrt{2} \frac{\kappa h_{11} v_{u}^{2}}{m} ; m_{\nu_{3}} \simeq \frac{\kappa^{2} v_{u}^{2}}{2 m}\left(\frac{\varepsilon_{m}}{m}\right), \\
\Delta m_{12}^{2} & \simeq \frac{\kappa^{3} h_{11} v_{u}^{4}}{m^{2}}\left(\frac{\varepsilon_{m}}{m}\right) \quad \text { and } \quad \Delta m_{23}^{2} \simeq \frac{2 \kappa^{2} h_{11}^{2} v_{u}^{4}}{m^{2}},
\end{aligned}
$$

\footnotetext{
${ }^{5}$ Even with the new $\mathrm{Z}_{4}^{\prime}, \frac{\left(z_{3} N_{1}\right)^{4} z_{1} N_{1} N_{2}}{M_{P}^{8}}$ term will be allowed, however this term turns out to be very small.
} 
where $h_{22} \simeq h_{23}=\kappa$ is assumed for simplicity and $\left\langle H_{u}\right\rangle=v_{u}$. It also indicates a bi-maximal mixing pattern in solar and atmospheric sectors along with $\theta_{13} \simeq \frac{\varepsilon_{m}}{m} \frac{\kappa}{h_{11}}$.

Now as $m$ is essentially determined from the inflation part in our scenario, we find $h_{11}$ of order $\sim \mathcal{O}\left(10^{-2}\right)$ to get correct magnitude of $\Delta m_{23}^{2} \simeq 2.5 \times 10^{-3} \mathrm{eV}^{2}[36]$ with $v_{u}=174 \mathrm{GeV}$ and $\kappa=1$. At first sight it is tantalizing to note that with $\frac{\varepsilon_{m}}{m} \ll 1$ and we could also accommodate $\Delta m_{12}^{2}\left(\simeq 7 \times 10^{-5} \mathrm{eV}^{2}[36]\right)$. However $\frac{\varepsilon_{m}}{m} \simeq \frac{\beta \Lambda}{m} \frac{\operatorname{Tr}\langle\Phi\rangle}{M_{P}} \sim \frac{\mu}{M_{P}} \leq \mathcal{O}\left(10^{-6}\right)$ and it turns out to be too small (a value of $\frac{\varepsilon_{m}}{m} \sim 10^{-2}$ could fulfill the requirement) to explain the solar splitting correctly. Therefore small but relatively larger entries are required in place of $\left(\frac{\varepsilon_{m}}{m}\right)$ terms in $m_{\nu}$ [37]. A possible source of these terms could arise in our case from higher order R-symmetry breaking terms. The mixing angles $\theta_{12}, \theta_{13}$ can be corrected from the contribution in the charged lepton sector. We do not explore this possibility in detail here. It could as well be the effect of renormalization group evolutions as pointed out by [37], or even other sources (e.g. type-II contribution as in [38]) of neutrino mass.

\section{Reheating}

As soon as Hubble parameter becomes less than the mass of the inflaton, $N_{1}$ starts to oscillate around its minimum and universe reheats. The estimate of $h$ helps us determining the reheat temperature. The decay of $N_{1}$ is governed through the $W$ in eq. (9.1). The decay width therefore is estimated to be

$$
\Gamma_{N_{1}}=\frac{\left(2 \kappa^{2}+h_{11}^{2}\right)}{8 \pi} m
$$

neglecting the effect of $\varepsilon_{m}$ term. The corresponding reheat temperature is obtained as

$$
T_{R H}=\left(\frac{45}{2 \pi^{2} g_{*}}\right)^{1 / 4} \sqrt{\Gamma_{N_{1}} M_{P}} \simeq 4 \times 10^{14} \mathrm{GeV},
$$

where $m \sim 10^{-6} M_{P}$ is considered and $\kappa \sim \mathcal{O}(1)$, as obtained from the discussion of the previous section. Such a high reheating temperature poses a threat in terms of over abundance of thermally produced gravitinos. ${ }^{6}$ Their abundance is mostly proportional to the reheat temperature [41],

$$
Y_{3 / 2} \simeq 2 \times 10^{-9}\left(\frac{T_{R H}}{10^{13} \mathrm{GeV}}\right)
$$

where $Y_{3 / 2}=\frac{n_{3 / 2}}{s}$ with $n_{3 / 2}$ as the number density of gravitinos and $s$ is the entropy density. These gravitinos, if massive, then decays into the lightest supersymmetric particles (LSP) and can destroy the predictions of primordial abundance of light elements. On the other hand, if gravitino is the LSP, the reheating temperature can not be as high as mentioned in our work. This problem can be circumvented if the gravitinos are superlight, e.g. $m_{3 / 2} \sim 16 \mathrm{eV}$ [42]. Such a gravitino can be accommodated in the gauge mediated supersymmetry breaking. In our set-up, $\mu$ is the scale which in turn predicts the gravitino

\footnotetext{
${ }^{6}$ Note that the chaotic inflation is free from gravitino problem indeed for the non-thermal decay of inflaton [39, 40].
} 
mass through $m_{3 / 2} \simeq \frac{\mu^{2}}{\sqrt{3} M_{P}}$. Therefore with $\mu \sim 10^{5} \mathrm{GeV}$, such a light gravitino mass can be obtained. Another way to circumvent this gravitino problem is through the late time entropy production [43]. Apart from these possibilities one interesting observation by [44] could be of help in this regard. The author in [44] have shown that once the messenger mass scale (in case of gauge mediation of supersymmetry breaking) falls below the reheat temperature, the relic abundance of thermally produced gravitinos becomes insensitive to $T_{R H}$ and a large $T_{R H} \sim 10^{13-14} \mathrm{GeV}$ can be realized.

Finally we make brief comments on leptogenesis in the present scenario. Considering $m_{3} \ll m, N_{3}$ would contribute mostly for the lepton asymmetry production. The CP asymmetry generated can be estimated as [45]

$$
\epsilon_{3}=\frac{3}{8 \pi v_{u}^{2}} \frac{1}{\left(\hat{m}_{D}^{\dagger} \hat{m}_{D}\right)_{33}} \sum_{i=1,2} \operatorname{Im}\left[\left(\hat{m}_{D}^{\dagger} \hat{m}_{D}\right)_{i 3}^{2}\right] \frac{m_{3}}{m} .
$$

Here $\hat{m_{D}}$ represents the rotated Dirac mass matrix in the basis where $M_{R}$ is diagonal. It is found that CP-asymmetry exactly vanishes in this case. We expect this can be cured with the introduction of higher order $\mathrm{U}(1)_{R}$ symmetry breaking terms which could be introduced into $m_{D}$ and $M_{R} \cdot{ }^{7}$ Then similar to [46], a non-zero lepton asymmetry through the decay of $N_{3}$ can be realized.

\section{Conclusion}

We have considered the superpartner of a right-handed neutrino as playing the role of inflaton. Although a minimal chaotic inflation scenario out of this consideration is a well studied subject, its simplest form is almost outside the $2 \sigma$ region of recent $n_{s}-r$ plot by PLANCK 2015. We have shown in this work, that a mere coupling with the SQCD sector responsible for supersymmetry breaking can be considered as a deformation to the minimal version of the chaotic inflation. Such a deformation results in a successful entry of the chaotic inflation into the latest $n_{s}-r$ plot. Apart from this, the construction also ensures that a remnant supersymmetry breaking is realized at the end of inflation. The global $\mathrm{U}(1)_{R}$ symmetry plays important role in constructing the superpotential for both the RH neutrino as well as SQCD sector. We have shown that the shift-symmetry breaking terms in the set up can be accommodated in an elegant way by introducing spurions. Their introduction, although ad hoc, can not only explain the size of the symmetry breaking but also provide a prescription for operators involving the $\mathrm{RH}$ neutrino superfields (responsible for inflation) in the superpotential. With the help of the $R$-symmetry and the discrete symmetries introduced, we are able to show that light neutrino masses and mixing resulted from the set-up can accommodate the recent available data nicely, predicting an inverted hierarchy for light neutrinos. However there still exists a scope for further study in terms of leptogenesis through the $R$-symmetry breaking terms.

\footnotetext{
${ }^{7}$ We have already mentioned about this possibility of inclusion of such (small) term in the previous section, that can correct the $\Delta m_{12}^{2}$ and the lepton mixing angles.
} 


\section{A Finding the root of $\sigma$}

Setting $\frac{\partial V_{\operatorname{Inf}}(\tilde{\chi}, \tilde{\sigma})}{\partial \tilde{\sigma}}=0$, we get a fifth order polynomial equation in $\tilde{\sigma}$ of the form,

$$
\frac{\tilde{\sigma}^{5}}{2}+\tilde{\sigma}^{3}+k_{1}\left[1-\frac{6(1-p)}{\tilde{\chi}^{2}}+\tilde{\sigma}^{2}\left\{\frac{1}{4}-\frac{6(1-p)}{\tilde{\chi}^{2}}\right\}-\frac{\tilde{\sigma}^{4}}{4}\right]+k_{2} \tilde{\sigma}=0,
$$

where $k_{1}=-\frac{2 A}{\tilde{m}^{2}} \tilde{\chi}^{5-6 p}$ and $k_{2}=\frac{4}{\tilde{\chi}^{2}}\left[1+\tilde{\chi}^{10-12 p} \frac{A^{2}}{2 \tilde{m}^{4}}\left\{9(1-p)^{2}-\frac{\tilde{\chi}^{2}}{4}\right\}\right]$. Here we disregard the first and third terms from the coefficient of $\tilde{\sigma}^{3}$ in eq. (5.4) as $\tilde{\chi}$ being greater than one during inflation, $\frac{\tilde{m}^{2} \tilde{\chi}^{2}}{4}$ is the dominant contribution. We now try to solve the eq. (A.1) to express $\langle\tilde{\sigma}\rangle$ in terms of $\tilde{\chi}$. In doing so, note that $\tilde{\chi}$ being inflaton is super-Planckian while $\tilde{\sigma}$ remains sub-Planckian $(\tilde{\sigma}<1)$ during inflation. Also the parameters involved, $\tilde{\Lambda}$ and $\tilde{m}$, are considered to be much less than one (in $M_{P}$ unit), $\tilde{\Lambda}, \tilde{m} \ll 1$ with $\tilde{\Lambda} \tilde{\chi} \ll 1$. We have also taken $\tilde{\Lambda} \geq \tilde{m}$. Since the added contribution via $W_{\text {Int }}$ is expected to provide modification only on the minimal chaotic inflation, it is natural that $m$ should be close to $10^{13} \mathrm{GeV}$ (also $\tilde{\chi}$ is expected of order $\mathcal{O}(10)$ ). These consideration keeps $k_{1}$ to be less than one $\left(k_{1}<1\right)$ although $k_{2}$ can be somewhat larger.

With $p=4 / 7$, we find $\tilde{\sigma}^{4}$ can be neglected and the eq. (A.1) then reduces to the form

$$
\frac{\tilde{\sigma}^{5}}{2}+\left(\tilde{\sigma}^{3}+c_{1} \tilde{\sigma}^{2}+k_{2} \tilde{\sigma}+c_{3}\right)=0
$$

where $c_{1}=k_{1}\left(\frac{1}{4}-\frac{18}{7 \tilde{\chi}^{2}}\right)$ and $c_{3}=k_{1}\left(1-\frac{18}{7 \tilde{\chi}^{2}}\right)$. The coefficient of $\tilde{\sigma}^{5}$ being $1 / 2$, the $\tilde{\sigma}^{5}$ term can be considered as a perturbation over the cubic equation in $\tilde{\sigma}$, as indicated by the first brackets in eq. (A.2). Let $\tilde{\sigma_{0}}$ be the solution of this cubic part of eq. (A.2) and the analytic form of it can easily be obtained (for real root). Then we consider the solution of eq. (A.2) as

$$
\tilde{\sigma}=\tilde{\sigma}_{0}+\epsilon \tilde{\sigma}_{1}+\epsilon^{2} \tilde{\sigma}_{2}+\epsilon^{3} \tilde{\sigma}_{3},
$$

with $\epsilon=1 / 2$ (coefficient of $\tilde{\sigma}_{5}$ term) as a perturbation parameter. Finally we get

$$
\begin{aligned}
& \tilde{\sigma}_{1}=\frac{-\tilde{\sigma}_{0}^{5}}{k_{2}+2 c_{1} \tilde{\sigma}_{0}+3 \tilde{\sigma}_{0}^{2}}, \\
& \tilde{\sigma}_{2}=\frac{-5 \tilde{\sigma}_{0}^{4} \tilde{\sigma}_{1}-3 \tilde{\sigma}_{0} \tilde{\sigma}_{1}^{2}-c_{1} \tilde{\sigma}_{1}^{2}}{k_{2}+2 c_{1} \tilde{\sigma}_{0}+3 \tilde{\sigma}_{0}^{2}}, \\
& \tilde{\sigma}_{3}=\frac{-5 \tilde{\sigma}_{0}^{4} \tilde{\sigma}_{2}-10 \tilde{\sigma}_{0}^{3} \tilde{\sigma}_{1}^{2}-6 \tilde{\sigma}_{0} \tilde{\sigma}_{1} \tilde{\sigma}_{2}-\tilde{\sigma}_{1}^{3}-2 c_{1} \tilde{\sigma}_{1} \tilde{\sigma}_{2}}{k_{2}+2 c_{1} \tilde{\sigma}_{0}+3 \tilde{\sigma}_{0}^{2}} .
\end{aligned}
$$

We have checked numerically (using mathematica) that this perturbation method for solving the fifth order polynomial equation as in eq. (A.1) works reasonably well. For comparison, we have included figure 3 where $\langle\tilde{\sigma}\rangle$ is depicted against the variation of $\tilde{\chi}$ (particularly during inflation when $\chi$ acquires super-Planckian value). The solid line represents the VEV of $\tilde{\sigma}$ as obtained from our perturbation method and the dashed line gives the exact numerical estimate of $\langle\tilde{\sigma}\rangle$ from eq. (A.1). In order to get $V_{\text {Inf }}$ in terms of $\tilde{\chi}$, we have used the analytic form of $\langle\tilde{\sigma}\rangle$ obtained through this perturbation method. 


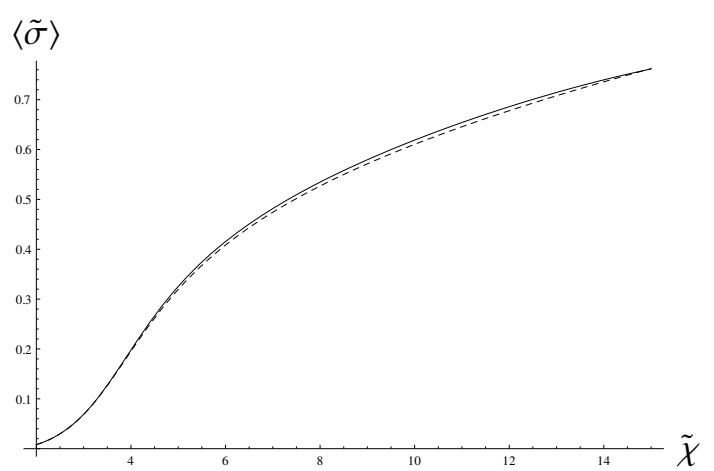

Figure 3. Comparison of $\langle\tilde{\sigma}\rangle$ with $\tilde{\chi}$ using perturbation (solid line) and exact numerical result (dashed line).

\begin{tabular}{|l|l|l|l|}
\hline Fields & $\mathrm{U}(1)_{B}$ & $\mathrm{U}(1)_{A}$ & $\mathrm{U}(1)_{R^{\prime}}$ \\
\hline $\mathrm{Q}$ & 1 & 1 & $\frac{2}{9}$ \\
$\tilde{Q}$ & -1 & 1 & $\frac{2}{9}$ \\
\hline$\Lambda$ & 0 & $\frac{3}{2}$ & 0 \\
$W$ & 0 & 0 & 2 \\
\hline$q$ & $\frac{7}{2}$ & $-\frac{1}{4}$ & $\frac{7}{9}$ \\
$\tilde{q}$ & $-\frac{7}{2}$ & $-\frac{1}{4}$ & $\frac{7}{9}$ \\
$\Phi$ & 0 & $\frac{1}{2}$ & $\frac{4}{9}$ \\
\hline
\end{tabular}

Table 5. Global charges of various fields in a massless SQCD theory [30].

\section{B $\quad R$ charges of various fields}

Here we discuss the $R$-charge assignments for the various fields involved in our construction. Firstly in table 5, we include various U(1) global charges associated with massless SQCD theory $\left(N_{f}=9, N_{c}=7\right)$ following [30]. However once the term $m_{Q} \operatorname{Tr} Q \tilde{Q}$ is included in the UV description and a baryonic deformation (through $m_{q} q q$ term in eq. (8.1)) is considered as well in the magnetic description, there exists a residual $\mathrm{U}(1)_{R}$ symmetry only. The charges of the fields in the magnetic description can be obtained [47] from

$$
R=\frac{2}{7} B+\frac{28}{9} A+R^{\prime}
$$

This redefined R-charges are mentioned in table 3. The superpotential in eq. (9.1) respects this $\mathrm{U}(1)_{R}$ symmetry. From $\Phi=\operatorname{Tr}(Q \tilde{Q}) / \Lambda$, the $Q \tilde{Q}$ combination has two units of $R$ charges.

Open Access. This article is distributed under the terms of the Creative Commons Attribution License (CC-BY 4.0), which permits any use, distribution and reproduction in any medium, provided the original author(s) and source are credited. 


\section{References}

[1] BICEP2 collaboration, P.A.R. Ade et al., Detection of B-mode polarization at degree angular scales by BICEP2, Phys. Rev. Lett. 112 (2014) 241101 [arXiv:1403.3985] [INSPIRE].

[2] Planck collaboration, P.A.R. Ade et al., Planck 2015 results. XIII. Cosmological parameters, arXiv: 1502.01589 [INSPIRE].

[3] Planck collaboration, P.A.R. Ade et al., Planck 2015 results. XX. Constraints on inflation, arXiv: 1502.02114 [INSPIRE].

[4] M. Kawasaki, M. Yamaguchi and T. Yanagida, Natural chaotic inflation in supergravity, Phys. Rev. Lett. 85 (2000) 3572 [hep-ph/0004243] [InSPIRE].

[5] M. Kawasaki, M. Yamaguchi and T. Yanagida, Natural chaotic inflation in supergravity and leptogenesis, Phys. Rev. D 63 (2001) 103514 [hep-ph/0011104] [INSPIRE].

[6] A.D. Linde, Inflationary cosmology, Lect. Notes Phys. 738 (2008) 1 [arXiv:0705.0164] [INSPIRE].

[7] K. Harigaya, M. Kawasaki and T.T. Yanagida, Lower bound of the tensor-to-scalar ratio $r \gtrsim 0.1$ in a nearly quadratic chaotic inflation model in supergravity, Phys. Lett. B 741 (2015) 267 [arXiv:1410.7163] [INSPIRE].

[8] T. Li, Z. Li and D.V. Nanopoulos, Supergravity inflation with broken shift symmetry and large tensor-to-scalar ratio, JCAP 02 (2014) 028 [arXiv:1311.6770] [INSPIRE].

[9] Planck collaboration, P.A.R. Ade et al., Planck 2013 results. XVI. Cosmological parameters, Astron. Astrophys. 571 (2014) A16 [arXiv:1303.5076] [INSPIRE].

[10] K. Nakayama, F. Takahashi and T.T. Yanagida, Polynomial chaotic inflation in supergravity, JCAP 08 (2013) 038 [arXiv: 1305.5099] [INSPIRE].

[11] K. Nakayama, F. Takahashi and T.T. Yanagida, Polynomial chaotic inflation in supergravity revisited, Phys. Lett. B 737 (2014) 151 [arXiv:1407.7082] [INSPIRE].

[12] H. Murayama, H. Suzuki, T. Yanagida and J. Yokoyama, Chaotic inflation and baryogenesis by right-handed sneutrinos, Phys. Rev. Lett. 70 (1993) 1912 [INSPIRE].

[13] J.R. Ellis, Sneutrino inflation, Nucl. Phys. Proc. Suppl. 137 (2004) 190 [hep-ph/0403247] [INSPIRE].

[14] S. Khalil and A. Sil, Right-handed sneutrino inflation in SUSY B-L with inverse seesaw, Phys. Rev. D 84 (2011) 103511 [arXiv:1108.1973] [INSPIRE].

[15] H. Murayama, K. Nakayama, F. Takahashi and T.T. Yanagida, Sneutrino chaotic inflation and landscape, Phys. Lett. B 738 (2014) 196 [arXiv:1404.3857] [INSPIRE].

[16] J.L. Evans, T. Gherghetta and M. Peloso, Affleck-Dine sneutrino inflation, Phys. Rev. D 92 (2015) 021303 [arXiv: 1501.06560] [INSPIRE].

[17] K.A. Intriligator, N. Seiberg and D. Shih, Dynamical SUSY breaking in meta-stable vacua, JHEP 04 (2006) 021 [hep-th/0602239] [INSPIRE].

[18] P. Brax, C.A. Savoy and A. Sil, Intermediate scale inflation and metastable supersymmetry breaking, Phys. Lett. B 671 (2009) 374 [arXiv:0807.1569] [InSPIRE].

[19] C.A. Savoy and A. Sil, Can inflation induce supersymmetry breaking in a metastable vacuum?, Phys. Lett. B 660 (2008) 236 [arXiv:0709.1923] [INSPIRE]. 
[20] P. Brax, C.A. Savoy and A. Sil, SQCD inflation \& SUSY breaking, JHEP 04 (2009) 092 [arXiv: 0902.0972] [INSPIRE].

[21] N.J. Craig, ISS-flation, JHEP 02 (2008) 059 [arXiv:0801.2157] [INSPIRE].

[22] S. Abel, C. Durnford, J. Jaeckel and V.V. Khoze, Dynamical breaking of U(1)(R) and supersymmetry in a metastable vacuum, Phys. Lett. B 661 (2008) 201 [arXiv:0707.2958] [INSPIRE].

[23] G. 't Hooft et al., Recent developments in gauge theories, NATO Sci. Ser. B 59 (1980) 1.

[24] K. Harigaya, M. Ibe, M. Kawasaki and T.T. Yanagida, Revisiting the minimal chaotic inflation model, arXiv:1506.05250 [INSPIRE].

[25] C. Pallis, Kinetically modified nonminimal chaotic inflation, Phys. Rev. D 91 (2015) 123508 [arXiv: 1503.05887] [INSPIRE].

[26] G. Barenboim and W.-I. Park, New- vs. chaotic-inflations, arXiv:1504.02080 [InSPIRE].

[27] L.M. Carpenter and S. Raby, Chaotic hybrid inflation with a gauged B-L, Phys. Lett. B 738 (2014) 109 [arXiv: 1405.6143] [INSPIRE].

[28] L. Heurtier, S. Khalil and A. Moursy, Single field inflation in supergravity with a U(1) gauge symmetry, JCAP 10 (2015) 045 [arXiv:1505.07366] [INSPIRE].

[29] W. Buchmüller, E. Dudas, L. Heurtier and C. Wieck, Large-field inflation and supersymmetry breaking, JHEP 09 (2014) 053 [arXiv: 1407.0253] [INSPIRE].

[30] K.A. Intriligator and N. Seiberg, Lectures on supersymmetry breaking, Class. Quant. Grav. 24 (2007) S741 [hep-ph/0702069] [INSPIRE].

[31] K. Harigaya, M. Ibe, K. Schmitz and T.T. Yanagida, Dynamical fractional chaotic inflation, Phys. Rev. D 90 (2014) 123524 [arXiv:1407.3084] [InSPIRE].

[32] K. Harigaya, M. Ibe, K. Schmitz and T.T. Yanagida, Dynamical chaotic inflation in the light of BICEP2, Phys. Lett. B 733 (2014) 283 [arXiv:1403.4536] [INSPIRE].

[33] X. Gao, T. Li and P. Shukla, Fractional chaotic inflation in the lights of PLANCK and BICEP2, Phys. Lett. B 738 (2014) 412 [arXiv:1404.5230] [INSPIRE].

[34] R.N. Mohapatra and G. Senjanović, Neutrino mass and spontaneous parity violation, Phys. Rev. Lett. 44 (1980) 912 [INSPIRE].

[35] R. Barbieri, L.J. Hall, D. Tucker-Smith, A. Strumia and N. Weiner, Oscillations of solar and atmospheric neutrinos, JHEP 12 (1998) 017 [hep-ph/9807235] [INSPIRE].

[36] Particle Data Group collaboration, K.A. Olive et al., Review of particle physics, Chin. Phys. C 38 (2014) 090001 [inSPIRE].

[37] K.S. Babu and R.N. Mohapatra, Predictive schemes for bimaximal neutrino mixings, Phys. Lett. B 532 (2002) 77 [hep-ph/0201176] [INSPIRE].

[38] B. Karmakar and A. Sil, Spontaneous CP-violation in Lepton-sector: a common origin for $\theta_{13}$, Dirac CP phase and leptogenesis, arXiv:1509.07090 [INSPIRE].

[39] M. Kawasaki, F. Takahashi and T.T. Yanagida, The gravitino-overproduction problem in inflationary universe, Phys. Rev. D 74 (2006) 043519 [hep-ph/0605297] [INSPIRE].

[40] M. Kawasaki, F. Takahashi and T.T. Yanagida, Gravitino overproduction in inflaton decay, Phys. Lett. B 638 (2006) 8 [hep-ph/0603265] [INSPIRE]. 
[41] M. Kawasaki, K. Kohri, T. Moroi and A. Yotsuyanagi, Big-bang nucleosynthesis and gravitino, Phys. Rev. D 78 (2008) 065011 [arXiv:0804.3745] [INSPIRE].

[42] M. Viel, J. Lesgourgues, M.G. Haehnelt, S. Matarrese and A. Riotto, Constraining warm dark matter candidates including sterile neutrinos and light gravitinos with WMAP and the Lyman-alpha forest, Phys. Rev. D 71 (2005) 063534 [astro-ph/0501562] [INSPIRE].

[43] K. Kohri, M. Yamaguchi and J. Yokoyama, Production and dilution of gravitinos by modulus decay, Phys. Rev. D 70 (2004) 043522 [hep-ph/0403043] [INSPIRE].

[44] H. Fukushima and R. Kitano, Gravitino thermal production revisited and a new cosmological scenario of gauge mediation, JHEP 01 (2014) 081 [arXiv:1311.6228] [INSPIRE].

[45] W. Buchmüller and T. Yanagida, Quark lepton mass hierarchies and the baryon asymmetry, Phys. Lett. B 445 (1999) 399 [hep-ph/9810308] [INSPIRE].

[46] E.E. Jenkins and A.V. Manohar, Tribimaximal mixing, leptogenesis and $\theta_{13}$, Phys. Lett. B 668 (2008) 210 [arXiv:0807.4176] [INSPIRE].

[47] C. Durnford, Duality and models of supersymmetry breaking, PhD. thesis, Durham University, Durham, U.K. (2009). 